\title{
A New Performance Bound for PAM-Based CPM Detectors
}

\author{
Erik Perrins, Member, IEEE, and Michael Rice, Senior Member, IEEE
}

\begin{abstract}
It is well understood that the pulse amplitude modulation (PAM) representation of continuous phase modulation (CPM) can lead to reduced-complexity detectors with near optimum performance. It has recently been shown that the PAM representation also extends to CPM schemes with multiple modulation indexes (multi- $h$ CPM). In this paper, we present a detector for multi- $h$ CPM which is based on the PAM representation. We also give an exact expression for the pairwise error probability for the entire class of PAM-based CPM detectors (single- and multi- $h$, optimal, and reduced-complexity) over the additive white Gaussian noise (AWGN) channel and show that this bound is tighter than the previously published bound for approximate PAM-based detectors. In arriving at this expression, we show that PAM-based detectors for CPM are a special case of the broad class of mismatched CPM detectors. We also show that the metrics for PAM-based detectors accumulate distance in a different manner than metrics for other CPM detectors. These distance properties are especially useful in applications with greatly reduced trellis sizes. We give thorough examples of the analysis for different single- and multi- $h$ signaling schemes. We also apply the new bound in comparing the performance of PAM-based detectors with other reduced-complexity detectors for CPM.
\end{abstract}

Index Terms-Continuous phase modulation (CPM), mismatched detector, pairwise error probability, pulse amplitude modulation (PAM), reduced-complexity detector, union bound.

\section{INTRODUCTION}

C ONTINUOUS phase modulation (CPM) is advantageous for its efficient use of power and bandwidth. It also has a constant signal envelope, which is essential in applications using nonlinear amplifiers. However, the optimal maximumlikelihood sequence detection (MLSD) scheme, which is implemented via the Viterbi algorithm (VA), often suffers from high complexity in terms of the required number of correlators (matched filters or MFs) and trellis states. A number of techniques have been proposed to reduce the number of MFs, e.g., [1]-[4], and similarly to reduce the number of trellis states, e.g., [1], [5]-[7].

Of particular interest here is the PAM representation of CPM, which was introduced by Laurent in 1986 [8]. In his paper, Laurent showed that any binary single- $h$ CPM scheme can be ex-

Paper approved by C. Tellambura, the Editor for Modulation and Signal Design of the IEEE Communications Society. Manuscript received October 12, 2004; revised March 8, 2005. This paper was presented in part at the IEEE Military Communications Conference, Monterey, CA, November 2004.

E. Perrins was with the Department of Electrical and Computer Engineering, Brigham Young University, Provo, UT 84602 USA. He is now with the Department of Electrical Engineering and Computer Science, University of Kansas, Lawrence, KS 66045 USA (e-mail: esp@ieee.org).

M. Rice is with the Department of Electrical and Computer Engineering, Brigham Young University, Provo, UT 84602 USA (e-mail: mdr@ee.byu.edu). Digital Object Identifier 10.1109/TCOMM.2005.857133 actly represented by a superposition of PAM waveforms. He also showed that the binary single- $h$ CPM signal is often well approximated by a reduced number of PAM pulses or even by the main pulse alone. Kaleh followed in 1989 [2] by deriving the MLSD structure for PAM-based CPM detectors. He also showed that suboptimal PAM-based detectors require an appreciably reduced number of MFs (since they are based on a limited number of pulses) and that they simultaneously achieve a reduction in the number of trellis states. Kaleh also provided a simple performance bound, which is of little use beyond the binary single- $h$ CPM schemes considered in his paper.

The PAM representation of CPM has since been extended to $M$-ary signaling by Mengali and Morelli [9] and for the special case of CPM schemes with integer modulation index by Huang and $\mathrm{Li}$ [10]. It has also been confirmed in these cases that reduced-complexity PAM-based detectors achieve a simultaneous reduction in the number of MFs and trellis states with manageable performance tradeoffs [10], [11]. These performance assessments have been made using computer simulations. There is a need for analysis and explanation of the performance of PAM-based detectors in general, since the computer simulations do not reveal the reasons for the strong performance of PAM-based detectors.

The PAM representation has also been extended to $M$-ary multi- $h$ CPM very recently in [12]. In this paper, we take this recent extension of the PAM-based CPM model and apply it to the problem of detecting $M$-ary multi- $h$ CPM signals. We generalize Kaleh's results from [2] and arrive at an optimal MLSD structure for multi- $h$ CPM that is based on the PAM representation. We confirm that the MF and trellis reduction properties of suboptimal PAM-based detectors also hold for the multi- $h$ case.

One important facet of PAM-based detectors for CPM which is missing from [2], [11] is an adequate performance analysis of these detectors. We study the problem of performance in this paper and derive the exact expression for the pairwise error probability for PAM-based CPM detectors in AWGN. This pairwise error probability, though given in multi- $h$ terms, is also applicable to the entire class of PAM-based detectors for CPM in [2], [11] (both optimal and approximate). In carrying out this analysis, we show that detectors based on the PAM approximation can be viewed as mismatched CPM detectors. Schemes of this type, where the internal signal model of the detector is mismatched (different) with respect to the signal produced by the transmitter, were first analyzed in [1]. The class of mismatched detectors is quite broad and includes the schemes in e.g., [3], [4]. As such, the analysis presented here is a special case of that given in [1]. In order for this viewpoint to yield correct results, however, proper consideration is given to the precise 
manner in which metrics are computed in PAM-based detectors. We demonstrate that, like other mismatched detectors, the pairwise error probability is a function of specific pairs of data sequences, in contrast with the optimal detector where only the difference between pairs of data sequences is needed. This in turn means that the error performance for reduced-complexity PAM detectors is not dominated by a single distance parameter, i.e., the minimum distance.

We apply the new bound in a performance comparison between PAM-based detectors and the reduced complexity detectors given in [1] and [6]. In this comparison, we demonstrate explicitly how metrics within PAM-based detectors accumulate distance in a different manner than metrics in other CPM detectors. For instance, if two CPM signals are different from each other for a brief interval, the optimal detector observes the distance between these signals over this entire event. By contrast, a detector with a reduced trellis often observes the distance between these signals over some fraction of this interval (thus forfeiting some portion of the optimal distance). The reduced complexity scheme in [1] observes the (mismatched) distance during the center of the interval, splitting the omitted portion evenly between the beginning and the ending tails. The decision feedback scheme in [6] observes the distance from the beginning of the event to a certain point, discarding whatever remaining distance there is on the ending tail of the event. On the other hand, PAM-based detectors observe the (mismatched) distance from the beginning of the event to a certain point, after which they continue to observe the distance to a lesser degree up to the completion of the event. This behavior allows PAM-based detectors to be used with relatively small performance losses in spite of aggressively reduced trellis sizes. This study gives a theoretical basis to confirm the simulation results which have been reported for single- $h$ schemes in e.g., [2], [11].

In the next section, we review the traditional and PAM-based signal models for multi- $h$ CPM and give the structure of PAMbased detectors. In Section III, we analyze these detectors and obtain a new performance bound. In Section IV, we use the bound to characterize the performance of several single- and multi- $h$ schemes. We also apply the bound in a performance comparison in Section V and give conclusions in Section VI.

\section{MulTi- $h$ CPM Signal ModEL}

\section{A. Traditional Model}

The complex-baseband multi- $h$ CPM signal is given by

$$
\begin{aligned}
s(t, \boldsymbol{\alpha}) & =\sqrt{\frac{E}{T}} \exp (j \psi(t, \boldsymbol{\alpha})) \\
\psi(t, \boldsymbol{\alpha}) & =2 \pi \sum_{i=-\infty}^{n} \alpha_{i} h_{\underline{i}} q(t-i T), \quad n T \leq t<(n+1) T
\end{aligned}
$$

where $E$ is the symbol energy, $T$ is the symbol duration, $\left\{h_{\underline{i}}\right\}$ is the set of $N_{h}$ modulation indexes, $\boldsymbol{\alpha}=\left\{\alpha_{i}\right\}$ are the information symbols in the $M$-ary alphabet $\{ \pm 1, \pm 3, \ldots, \pm(M-1)\}$, and $q(t)$ is the phase pulse. In this paper, the underlined subscript notation in (2) is defined as modulo- $N_{h}$, i.e., $\underline{i} \triangleq i \bmod N_{h}$.
We assume the modulation indexes are rational numbers of the form [13]

$$
h_{\underline{i}}=\frac{k_{\underline{i}}}{p} .
$$

We determine $p$ by expressing all of the modulation indexes as a fraction and taking $p$ as the value of the smallest common denominator.

The phase pulse $q(t)$ is the integral of the frequency pulse $f(t)$. The frequency pulse is zero outside the time interval $(0, L T)$ and is scaled such that $q(L T)=1 / 2$. In light of these constraints on $f(t)$ and $q(t)$, and considering the $M$-ary digits $U_{n}=\left(\alpha_{n}+(M-1)\right) / 2$, (2) can be written as

$$
\psi(t, \boldsymbol{\alpha})=\theta\left(t, \boldsymbol{\alpha}_{n}\right)+\theta_{n-L}+\nu_{n}
$$

where

$$
\begin{aligned}
\theta\left(t, \boldsymbol{\alpha}_{n}\right) & =2 \pi \sum_{i=n-L+1}^{n} \alpha_{i} h_{\underline{i}} q(t-i T) \\
\theta_{n-L} & =\frac{2 \pi}{p}\left(\sum_{i=-\infty}^{n-L} U_{i} k_{\underline{i}}\right)_{\bmod p}
\end{aligned}
$$

and $\nu_{n}=\nu_{n-1}-\pi h_{\underline{n}}(M-1)$ is a data-independent phase tilt [13]. The term $\theta\left(t, \boldsymbol{\alpha}_{n}\right)$ is a function of the $L$ symbols being modulated by the phase pulse. The substitution of $U_{n}=\left(\alpha_{n}+\right.$ $(M-1)) / 2$ is confined to the phase state $\theta_{n-L}$, which takes on only $p$ distinct values. Therefore, the phase signal in (4) is described by a trellis containing $p M^{L-1}$ states, with $M$ branches at each state. Each branch is defined by the $(L+1)$-tuple

$$
\sigma_{n}=\left(\theta_{n-L}, \alpha_{n-L+1}, \ldots, \alpha_{n-1}, \alpha_{n}\right) .
$$

\section{B. PAM Model for Multi-h CPM}

In [12] it is shown that the right-hand side of (1) can be exactly written as

$$
s(t, \boldsymbol{\alpha})=\sqrt{\frac{E}{T}} \sum_{k=0}^{N-1} \sum_{n} a_{k, n} g_{k, \underline{n}}(t-n T)
$$

where $N=Q^{P}\left(2^{P}-1\right), Q=2^{L-1}$, and $P$ is the integer that satisfies the conditions $2^{P-1}<M \leq 2^{P}$. The signal is the superposition of pulses $g_{k, \underline{n}}(t)$ scaled by pseudo-symbols $a_{k, n}$. This is a generalization to the multi- $h$ case of the results in [8], [9] which apply to binary and $M$-ary single- $h$ CPM, respectively. The details of the construction of $\left\{g_{k, \underline{n}}(t)\right\}$ and $\left\{a_{k, n}\right\}$ are found in [12]. For this paper, it is important to know that the pulses $g_{k, \underline{n}}(t)$ vary in amplitude and duration, with the longest pulses also having the largest amplitude. This is shown in Fig. 1 for a quaternary CPM scheme with a raised-cosine frequency pulse of duration $3 T$ (3RC) and $h=\{4 / 16,5 / 16\}$. Each pulse has a duration of $D_{k}$ symbol times, where $D_{k}$ is an integer in the set $\{1,2, \ldots, L+1\}$. We can group the pulses into sets with common duration, i.e.,

$$
\mathcal{K}_{j}=\left\{k: D_{k}=L+1-j\right\}, \quad 0 \leq j \leq L .
$$



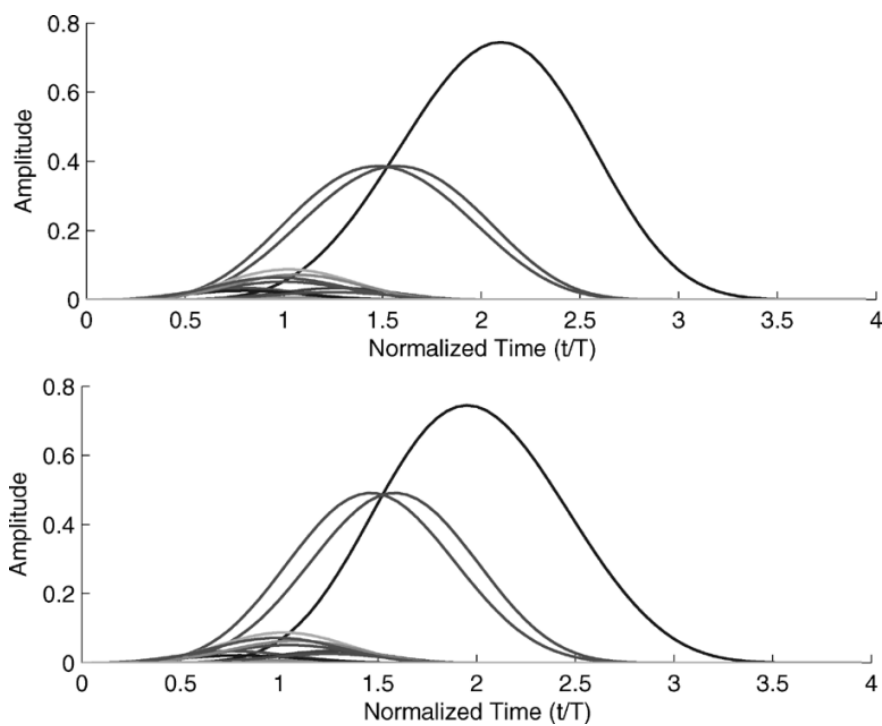

Fig. 1. Signal pulses $g_{k, \underline{n}}(t)$ for $M=4,3 R C$, with $h=\{4 / 16,5 / 16\}$. The 48 pulses for $n$-even are on top and the 48 for $n$-odd are on bottom. In each case there is one pulse of duration $4 T$, two of duration $3 T$, nine of duration $2 T$, and 36 of duration $T$ (too small to view in the figure).

Another important characteristic of the PAM representation is that not all the pseudo-symbols $a_{k, n}$ require the full $(L+$ 1)-tuple in (7) to describe them. We obtain a reduced trellis simply by discarding those signal terms that require the most states. In fact, it can be shown that the number of states required by the pseudo-symbols in each set $\mathcal{K}_{j}$ is $p M^{j-1}$, for $1 \leq j \leq L$ [14]. In order for this rule to be complete, we must also account for the case where $j=0$, i.e., the set $\mathcal{K}_{0}$, which we do by grouping $\mathcal{K}_{0}$ with $\mathcal{K}_{1}$, as was also done in [2] and [9]. In terms of Fig. 1, the first three pulses (those in $\mathcal{K}_{0}$ and $\mathcal{K}_{1}$ ) require a trellis with only $p M^{(1)-1}=16$ states. If the nine length- $2 T$ pulses in $\mathcal{K}_{2}$ are included then a trellis of $p M^{(2)-1}=64$ states is required. An additional $M$-fold increase in states is required if the 36 length- $T$ pulses are included. By simply discarding the smaller pulses and their costly pseudo-symbols, we see that the PAM representation simultaneously reduces the number of MFs and the number of trellis states. We stress that the principle behind the trellis reduction is a function of the pseudo-symbols and not the pulses. It just so happens that the structure is conveniently summarized in terms of the pulse duration.

The PAM model we will consider from this point on is

$$
\hat{s}(t, \boldsymbol{\alpha})=\sqrt{\frac{E}{T}} \sum_{k \in \mathcal{K}} \sum_{n} b_{k, n} c_{k, \underline{n}}(t-n T)
$$

where $\hat{s}(t, \boldsymbol{\alpha})$ is an approximation of the exact CPM signal $s(t, \boldsymbol{\alpha})$ in (8). The summation in (10) is over an arbitrary subset of signal terms $\mathcal{K}$, which is a proper subset of $\{0,1, \ldots, N-1\}$, and is usually chosen in terms of $\mathcal{K}_{j}$. The number of elements in the set is $|\mathcal{K}|$, and the shortest pulse duration is $D_{\min }=\min _{k \in \mathcal{K}} D_{k}$. The pulses $c_{k, \underline{n}}(t)$ and pseudo-symbols $b_{k, n}$ are related to the original pulses and pseudo-symbols by some averaging scheme. One such example is the minimum mean-squared error approximation in [8], [9], [12]. Another example is averaging the length-3T pulses in Fig. 1, which are all very similar, to produce a single pulse [11].

\section{PAM-Based Detectors for Multi-h CPM}

The optimal detector for the equivalent PAM representation of single- $h$ CPM was derived by Kaleh [2]. We now show the extension needed to accommodate the multi- $h$ case. The complex-baseband received signal model is

$$
r(t)=s(t, \boldsymbol{\alpha})+n(t)
$$

where $n(t)$ is a complex valued AWGN process with one-sided power spectral density $N_{0}$. Due to the AWGN assumption, the detector selects as its output the information sequence $\tilde{\boldsymbol{\alpha}}$ which minimizes the Euclidian distance

$$
\lambda(\tilde{\boldsymbol{\alpha}})=\int_{-\infty}^{\infty}|r(t)-s(t, \tilde{\boldsymbol{\alpha}})|^{2} d t .
$$

Since $s(t, \boldsymbol{\alpha})$ is constant envelope, minimizing (12) is equivalent to maximizing the correlation

$$
\lambda(\tilde{\boldsymbol{\alpha}})=\operatorname{Re} \int_{-\infty}^{\infty} r(t) s^{*}(t, \tilde{\boldsymbol{\alpha}}) d t
$$

which can be computed in a trellis using the recursive metric

$$
\lambda_{i}(n)=\lambda_{i}(n-1)+\operatorname{Re} \int_{n T}^{(n+1) T} r(t) s^{*}\left(t, \tilde{\boldsymbol{\alpha}}^{i}\right) d t
$$

where $\tilde{\boldsymbol{\alpha}}^{i}$ corresponds to the $(L+1)$-tuple associated with the $i$ th branch in the trellis and $\lambda_{i}(n)$ is the cumulative metric associated with $\tilde{\boldsymbol{\alpha}}^{i}$ at index $n$. We refer to (14) as the traditional detector metric and its performance is well understood [15].

To arrive at the PAM detector metric, we insert (10) into (12) and with some simple manipulations arrive at the recursion

$$
\lambda_{i}(n)=\lambda_{i}(n-1)+\operatorname{Re}\left\{\sum_{k \in \mathcal{K}} z_{k, \underline{n}} b_{k, \underline{n}}^{* i}\right\}-S^{i}
$$

where

$$
z_{k, \underline{n}}=\sqrt{\frac{E}{T}} \int_{n T}^{\left(n+D_{k}\right) T} r(t) c_{k, \underline{n}}(t-n T) d t
$$

is the output of a filter matched to $c_{k, n}(t)$ sampled at $t=(n+$ $\left.D_{k}\right) T$ and

$$
S^{i}=\frac{1}{2} \frac{E}{T} \int_{0}^{T}\left|\sum_{k \in \mathcal{K}} \sum_{n} b_{k, \underline{n}}^{\left(d_{i}, i\right)} c_{k, \underline{n}}(t-n T)\right|^{2} d t
$$

is a bias term that is a consequence of the approximation (10) no longer being constant envelope. The hypothesis along the $i$ th branch, $\tilde{\boldsymbol{\alpha}}^{i}$, is associated with a set of $|\mathcal{K}|$ branch pseudo-symbols $b_{k, n}^{i}$. A slightly different version of the branch pseudo-symbols $b_{k, \underline{n}}^{\left(\overline{d_{i}}, i\right)}$ is used (17). These are modified by decision feedback, as explained shortly.

We pause to discuss an important special case of (15) where the exact PAM representation in (8) is used. In other words, no approximations are made and $b_{k, n}=a_{k, n}, c_{k, \underline{n}}(t)=g_{k, \underline{n}}(t)$, and $|\mathcal{K}|=N$. Here the detector is based on a constant envelope signal. Therefore, the bias term is no longer necessary, since $S^{i}$ is a constant for all values of $i$. The correlation in (13) is computed exactly, the difference is that the computation is made using PAM pulses and pseudo-symbols rather than the traditional data-dependent CPM matched filters in (14). This special case represents an alternate form of MLSD and has equivalent performance to that of (14). 
The trellis for the PAM-based detector has $p M^{L^{\prime}-1}$ states, where

$$
L^{\prime}=L+1-D_{\min }
$$

We note that this reduced trellis is identical to those obtained from [1] and [6] ${ }^{1}$; the former reduces the trellis by basing the detector on a simpler (mismatched) CPM scheme, while the latter reduces the trellis with the use of decision feedback. In the present case, the trellis is reduced as a natural consequence of the PAM approximation. Each branch in the trellis has an $\left(L^{\prime}+1\right)$-tuple

$$
\sigma_{n}^{\prime}=\left(\theta_{n-L^{\prime}}, \alpha_{n-L^{\prime}+1}, \ldots, \alpha_{n-1}, \alpha_{n}\right)
$$

associated with it, and a corresponding set of $|\mathcal{K}|$ pseudo-symbols $b_{k, n}^{i}$. Since the interconnections (i.e., branches) in the multi- $h$ trellis vary in a modulo- $N_{h}$ fashion, we have added a modulo- $N_{h}$ index $\underline{n}$ to the branch pseudo-symbols $b_{k, \underline{n}}^{i}$. Practically speaking, the limits of integration in (16) mean the sampled MF outputs $z_{k, \underline{n}}$ are computed with a delay of $L$ symbol intervals, since $\max \left\{D_{k}\right\}=L+1$. This delay is directly related to the different manner in which the PAM metrics accumulate distance, as we shall see in the next section.

The bias term $S^{i}$ provides compensation for the signal energy variations present in the approximation. It presents a minor difficulty since it remains a function of the original $(L+1)$-tuple in (7). To cope with this, we use decision feedback where each state in the reduced trellis maintains a record of recent merge decisions $\left\{\hat{\alpha}_{n}\right\}$ which are used to fill out the original $(L+1)$-tuple. This minor use of decision feedback results in no performance loss (as we shall see in Section IV), which is consistent with other cases where decision feedback has been used, e.g., [6]. The record of recent decisions along the $i$-th branch is denoted by $d_{i}$ and the concatenation of $\left(d_{i}, i\right)$ in (17) forms an $(L+1)$-tuple. The bias term $S^{i}$ is not present in the detector configuration in [2], and can be ignored if (10) is very close to being constant envelope (as was the case in [2]); however, for more coarse PAM approximations, such as the multi- $h$ scheme discussed in Section IV, the removal of $S^{i}$ results in a surprisingly large performance penalty.

The structure of the detector is shown in Fig. 2. The received signal $r(t)$ is fed into the bank of $|\mathcal{K}|$ MFs. The sampled filter outputs $z_{k, \underline{n}}$ are the inputs to the VA, which computes branch metrics, determines the surviving path at each merging node, and outputs a decision. The figure also shows an expanded view of the $k$ th filter in the bank. Each filter actually consists of a set of $N_{h}$ filters whose sampled outputs are cyclically selected using a commutator and then delayed by the amount needed to have an overall filter delay of $L$ symbol times.

\section{Performance Analysis}

\section{A. Pairwise Error Probability for PAM-Based Detectors}

We seek the quantity $P\left(\boldsymbol{\alpha}_{i} \rightarrow \boldsymbol{\alpha}_{j}\right)$, which is the probability of the detector outputting the sequence $\boldsymbol{\alpha}_{j}$ given $\boldsymbol{\alpha}_{i}$ is transmitted. From (12), this pairwise error probability is

$$
P\left(\boldsymbol{\alpha}_{i} \rightarrow \boldsymbol{\alpha}_{j}\right)=\operatorname{Pr}\left\{\left\|r(t)-\hat{s}\left(t, \boldsymbol{\alpha}_{i}\right)\right\|^{2}>\left\|r(t)-\hat{s}\left(t, \boldsymbol{\alpha}_{j}\right)\right\|^{2}\right\}
$$

\footnotetext{
${ }^{1}$ We emphasize that the expressions for the branch metrics for each of these three detectors are each different from one another. The three detectors merely operate on an identical trellis structure.
}

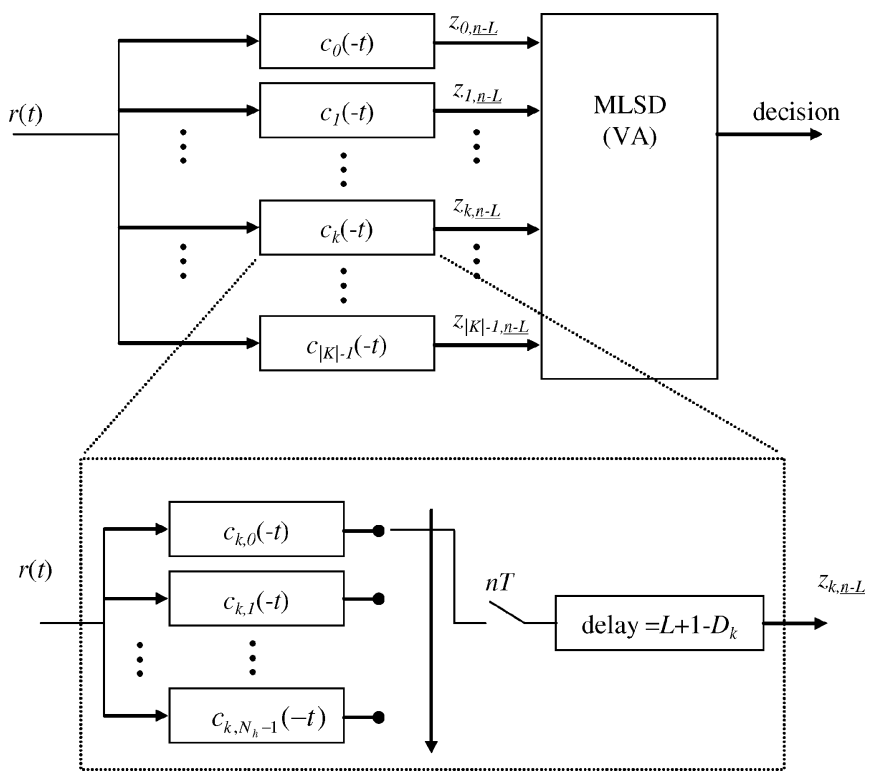

Fig. 2. PAM-based detector structure for multi- $h$ CPM with expanded view of modulo- $N_{h}$ matched filter and delay.

where

$$
\|x(t)\|^{2}=\langle x(t), x(t)\rangle, \quad\langle x(t), y(t)\rangle=\int_{-\infty}^{\infty} x(t) y^{*}(t) d t .
$$

While the limits of integration in (20) are infinite, the integrands are identical to each other (and thus cancel one another) except for the finite interval when the trellis paths taken by the two data sequences are different. If the data are different over a span of $R$ symbol times, then the two paths can merge together in the trellis after

$$
D_{\text {merger }}=R+L^{\prime}-1
$$

symbol times. We examine the branch metric in (15) over the interval $\left(N_{1} T, N_{2} T\right)$ where $N_{1}$ is arbitrary and $N_{2}=N_{1}+$ $D_{\text {merger. }}$. We concentrate on the first term in (15), which is a function of the received signal $r(t)$ and ignore $S^{i}$ for the moment. The PAM-based metric is

$$
\lambda_{i}\left(N_{1}, N_{2}\right)=\operatorname{Re} \int_{N_{1} T}^{\left(N_{2}+L\right) T} r(t) \tilde{s}^{*}\left(t, \boldsymbol{\alpha}_{i}, N_{1}, N_{2}\right) d t
$$

where the signal $\tilde{s}\left(t, \boldsymbol{\alpha}_{i}, N_{1}, N_{2}\right)$ is defined as

$$
\begin{aligned}
\tilde{s}(t, & \left.\boldsymbol{\alpha}_{i}, N_{1}, N_{2}\right) \\
& =\sqrt{\frac{E}{T}} \sum_{k \in \mathcal{K}} \sum_{m=\max \left(N_{1}, n-D_{k}+1\right)}^{\min \left(n, N_{2}-1\right)} b_{k, \underline{m}}^{i} c_{k, \underline{m}}(t-m T)
\end{aligned}
$$

for $n T \leq t<(n+1) T$. This signal $\tilde{s}\left(t, \boldsymbol{\alpha}_{i}, N_{1}, N_{2}\right)$ is key to understanding the performance of PAM-based detectors for CPM. Conceptually speaking, it is obtained by summing the outputs of a set of $|\mathcal{K}|$ filters with impulse response $c_{k, \underline{n}}(-t)$, $k \in\{\mathcal{K}\}$. These filters are idle (i.e., their output is zero) for $t<$ $N_{1} T$. During the $D_{\text {merger }}$ symbol times where the two trellis paths are different, these filters are fed with the pseudo-symbols $b_{k, \underline{n}}^{i}$ that correspond to the trellis path. After the paths merge, the filters are starved of input and their collective output returns 
to zero for $t \geq\left(N_{2}+L\right) T$. The cumbersome indexes on the inner sum in (24) ensure that pseudo-symbols are not clocked into the pulses for values of $n$ outside the range $n=N_{1}, N_{1}+$ $1, \ldots, N_{2}-1$.

We now include $S^{i}$ in the analysis. For convenience, we refer to $\hat{s}\left(t, \alpha_{i}\right)$ as $\hat{s}_{i}$ and $\tilde{s}\left(t, \boldsymbol{\alpha}_{i}, N_{1}, N_{2}\right)$ as $\tilde{s}_{i}$. We expand the terms in (20) in the same manner in which (15) was obtained from (12). By ,this we mean that the expansion includes a mix of $\hat{s}_{i}$ and $\tilde{s}_{i}$ terms, just as there are two terms found in (15). The pairwise error probability becomes

$P\left(\boldsymbol{\alpha}_{i} \rightarrow \boldsymbol{\alpha}_{j}\right)$

$=\operatorname{Pr}\left\{2 \operatorname{Re}\left\langle s_{i}, \tilde{s}_{i}-\tilde{s}_{j}\right\rangle+\left\|\hat{s}_{j}\right\|^{2}-\left\|\hat{s}_{i}\right\|^{2}<2 \operatorname{Re}\left\langle n(t), \tilde{s}_{j}-\tilde{s}_{i}\right\rangle\right\}$

which simplifies to

$$
P\left(\boldsymbol{\alpha}_{i} \rightarrow \boldsymbol{\alpha}_{j}\right)=Q\left(\sqrt{\frac{E_{b}}{N_{0}}} d^{\prime}(i, j)\right)
$$

where $E_{b}$ is the energy per bit $\left(E=E_{b} \log _{2} M\right)$ and

$$
\begin{aligned}
Q(x) & =\frac{1}{\sqrt{2 \pi}} \int_{x}^{\infty} e^{-u^{2} / 2} d u \\
d^{\prime}(i, j) & =\frac{d_{1}^{2}(i, j)}{d_{2}(i, j)} \\
d_{1}^{2}(i, j) & =\frac{\log _{2} M}{2 E}\left[2 \operatorname{Re}\left\langle s_{i}, \tilde{s}_{i}-\tilde{s}_{j}\right\rangle+\left\|\hat{s}_{j}\right\|^{2}-\left\|\hat{s}_{i}\right\|^{2}\right] \\
d_{2}^{2}(i, j) & =\frac{\log _{2} M}{2 E}\left\|\tilde{s}_{i}-\tilde{s}_{j}\right\|^{2} .
\end{aligned}
$$

We stress that (29) and (30) contain two forms of the approximate PAM signal. The received signal and noise are correlated with the modified signal $\tilde{s}_{i}$ and $\tilde{s}_{j}$ from (24). The branch bias terms $\hat{s}_{i}$ and $\hat{s}_{j}$ are from the traditional form of the PAM approximation in (10).

Some discussion of (28)-(30) is in order. While these expressions were derived from basic principles, they match the general form of the distance measure for mismatched CPM detectors in [1]. Here the transmitter/detector mismatch is due to the PAM approximation (i.e., discarding the less significant pulses and averaging the pulses). Other examples of mismatched signals are found in [1] and [4]. In light of this, the above analysis is a special case of the results in [1]. However, there are certain nuances regarding the PAM-based detector which must be given proper attention in order to obtain the correct result; namely, the mismatched signal must take the nonobvious form in (24) in order to to compute the distance measure correctly instead of its original form in (10). It can be shown that it is actually the reduced trellis itself (i.e., $L^{\prime}<L$ ), with its shortened merger duration in (22), which motivates the need for the modified signal in (24). It is also (24) which shows the unique manner by which distance is accumulated in the PAM-based detector. While the competing paths in the trellis are different for only $D_{\text {merger }}$ symbol intervals, the true CPM signals in (1) differ for $R+L-1$ symbol intervals (a difference of $L-L^{\prime}$ ). The limits on the integral in (16) show that, at index $n$, the distance measure has contributions from the received signal as far in advance as index $n+L$. Therefore, while (24) begins to decay after $D_{\text {merger }}$ symbol times, it does not return to zero until $L$ symbol times later. As such, distance not only continues to accumulate to a lesser degree, but this extra distance is applied retroactively at the time of the merge. As mentioned earlier, this attribute of the PAM-based CPM detector is distinct from those in [1] and [6].

We now make a final comment on (29). Although this is given as a squared quantity (for conceptual and historical reasons, e.g., [1]), there is nothing to prevent this quantity from assuming a negative value, especially as the PAM approximation (mismatch) becomes more coarse. In light of this, the final distance measure $d^{\prime}(i, j)$ is correctly used in unsquared form in the pairwise error probability (26) to preserve the sign. The case where $d^{\prime}(i, j)$ is negative corresponds to the detector outputting errors for arbitrarily large values of $E_{b} / N_{0}$ (i.e., an error floor).

\section{B. Probability of Bit Error}

Some additional steps are needed to convert the pairwise error probability into a probability of bit error. It is well known that, as $E_{b} / N_{0}$ grows large in the AWGN environment, a pairwise error probability term (26) corresponding to the minimum-distance becomes dominant [15]. This results from the nature of (27). The only question is at what point is $E_{b} / N_{0}$ large enough for this approximation to be accurate? ${ }^{2}$

For the optimal detector, there are many pairs of data sequences $\left(\boldsymbol{\alpha}_{i}, \boldsymbol{\alpha}_{j}\right)$ that have the minimum distance. Therefore, the pairwise error probability associated with the minimum distance is independent of specific pairs of $\left(\boldsymbol{\alpha}_{i}, \boldsymbol{\alpha}_{j}\right)$ and tends to dominate the union bound for $E_{b} / N_{0}$ of practical interest. In the case of the PAM-based detector (and other mismatched detectors), each specific $\left(\boldsymbol{\alpha}_{i}, \boldsymbol{\alpha}_{j}\right)$ pair has its own distance. Therefore, the pairwise error probability associated with the minimum distance is not typically dominant for $E_{b} / N_{0}$ of practical interest, since there are many near-minimum-distance terms also present in the union bound. In the case of the PAM-based detector, the probability of bit error is given by a sum of near-minimum-distance terms, as shown below. Examples illustrating this point are given in Section IV.

Based on the above arguments, the probability of bit error for the optimal detector is well approximated by

$$
P_{b} \approx \frac{N\left(\boldsymbol{\gamma}_{\min }\right) W\left(\boldsymbol{\gamma}_{\min }\right)}{N_{h} \cdot M^{R} \cdot \log _{2} M} Q\left(\sqrt{\frac{E_{b}}{N_{0}}} d_{\min }\right)
$$

We define the terms in (31) below. We start with the concept of a merger, which is a set of $\left(\boldsymbol{\alpha}_{i}, \boldsymbol{\alpha}_{j}\right)$ pairs with a common difference $\boldsymbol{\gamma}=\boldsymbol{\alpha}_{i}-\boldsymbol{\alpha}_{j}$ such that

$$
\left(\sum_{i} k_{\underline{i}} \frac{\gamma_{i}}{2}\right)_{\bmod p}=0
$$

where $\gamma_{i}$ is the $i$ th coordinate of $\gamma$, and $k_{i}$ and $p$ are from the modulation index $h_{\underline{i}}$ in (3). Equation (32) simply states that if two data sequences deviate from each other and their signals are to merge together at some later point, it must be that the summation of the difference in their phase is zero (modulo- $2 \pi$ ) when

\footnotetext{
${ }^{2}$ In this paper, we study the answer to this question in the context of uncoded CPM. The answer is different when considering the case of coded CPM, e.g., [16].
} 
properly scaled by the modulation indexes. In general, the difference sequence $\boldsymbol{\gamma}$ has nonzero coordinates that span a limited number of symbol times $(R)$ and we arbitrarily assign the first of these coordinates to be $\gamma_{0}$. We can easily count the number of pairs of data sequences $\left(\boldsymbol{\alpha}_{i}, \boldsymbol{\alpha}_{j}\right)$ with a common difference of $\pm \gamma$ as

$$
N(\boldsymbol{\gamma})=2 \prod_{l=0}^{R-1}\left(M-\frac{\left|\gamma_{l}\right|}{2}\right)
$$

Identifying the difference sequence $\boldsymbol{\gamma}_{\min }$ corresponding to the minimum-distance is a straightforward task for the optimal multi- $h$ CPM detector [15]. It is essentially to find the sequence

$$
\boldsymbol{\gamma}_{\min }=\arg \min _{\boldsymbol{\gamma}} d(\boldsymbol{\gamma})
$$

For multi- $h$ CPM, the search in (34) must be repeated $N_{h}$ times to allow each modulation index to coincide with $\gamma_{0}$. Also, since CPM is constant envelope, this distance does not vary across the $N\left(\boldsymbol{\gamma}_{\text {min }}\right)$ individual $\left(\boldsymbol{\alpha}_{i}, \boldsymbol{\alpha}_{j}\right)$ pairs in this merge.

We define $W(i, j)$ in (31) to be the difference in bits between a $\left(\boldsymbol{\alpha}_{i}, \boldsymbol{\alpha}_{j}\right)$ pair (i.e., the bit error weight), which is a function of the mapping from bits to symbols (typically a Gray code), and $\log _{2} M$ is the number of bits transmitted per use of the channel. The other terms in the denominator of (31) constitute $P\left(\boldsymbol{\alpha}_{i}\right)=1 /\left(N_{h} M^{R}\right)$, which is the probability that a given $M$-ary length- $R$ data sequence is transmitted with a particular alignment to the modulation indexes (we assume the uniform distribution).

For the PAM case, the minimum-distance merger itself is often the same $\boldsymbol{\gamma}_{\min }$ as the optimal detector, although there is nothing to prevent another merger from becoming dominant as the PAM approximation (mismatch) becomes more coarse. Therefore, the search over all mergers in (34) must be performed explicitly for the PAM distance measure in (28). Another difference from the optimal case, as was mentioned earlier, is that the distance in (28) is a function of specific sequence pairs $\left(\boldsymbol{\alpha}_{i}, \boldsymbol{\alpha}_{j}\right)$ and is different (in general) for each of the $N(\gamma)$ pairs in the merger. [This variation in distance is a general attribute of all mismatched CPM detectors; in the PAM case, it is caused by the missing or averaged pseudo-symbols in (10) that are still present in the exact signal in (8).] Modifying (31) accordingly produces

$$
P_{b} \approx \frac{W\left(\gamma_{\min }\right)}{N_{h} \cdot M^{R} \cdot \log _{2} M} \sum_{(i, j) \in \gamma_{\min }} Q\left(\sqrt{\frac{E_{b}}{N_{0}}} d^{\prime}(i, j)\right) .
$$

The scale factor of $N\left(\boldsymbol{\gamma}_{\min }\right)$ in (31) is replaced by a summation over all sequence pairs for which $\gamma=\gamma_{\text {min }}$. There will be a combination of $i, j$ that produces $d^{\prime}(i, j)=d_{\min }$; however, since this is only one of (potentially) many terms in the sum whose distances are relatively close to each other, it generally does not become dominant until $E_{b} / N_{0}$ is outside the range of practical interest. Therefore, PAM-based CPM detectors, generally speaking, are not well characterized by just one minimum-distance parameter as in (31); instead, the summation in (35) must be used.
TABLE I

Minimum-Distance MERGER PARAMETERS FOR THE EXAMPLES

\begin{tabular}{l|c|c|c|c}
\hline Example & $\boldsymbol{\gamma}_{\min }$ & $N\left(\boldsymbol{\gamma}_{\min }\right)$ & $W\left(\boldsymbol{\gamma}_{\min }\right)$ & $d_{\min }^{2}$ \\
\hline A. $3 R C$ & $\cdots, 0,2,-4,6,-4,2,0, \cdots$ & 72 & 7 & 1.29 \\
\hline B. $2 R C$ & $\cdots, 0,2,-2,0, \cdots$ & 18 & 2 & 1.33 \\
\hline C. $G M S K$ & $\cdots, 0,2,-2,0, \cdots$ & 2 & 2 & 1.69 \\
\hline
\end{tabular}

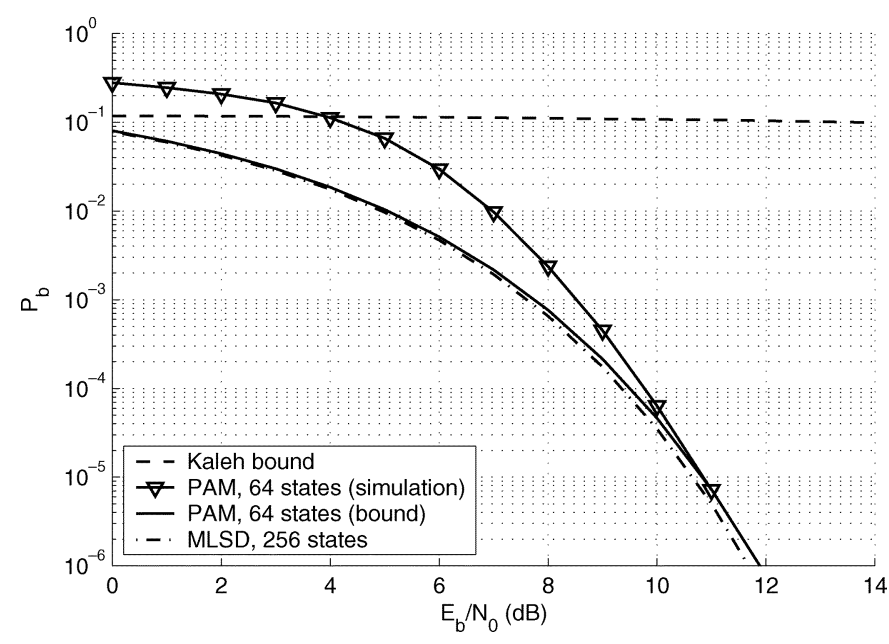

Fig. 3. Performance of $M=4,3 \mathrm{RC}$, with $h=\{4 / 16,5 / 16\}$. For the PAM detector, the $P_{b}$ curve shows strong agreement with the simulated data points. In this instance, the performance bound from Kaleh [2] essentially fails.

\section{EXAMPLES}

\section{A. Quaternary $3 R C$ With $h=\{4 / 16,5 / 16\}$}

The first example we consider is the multi- $h$ scheme $M=4$, 3RC (raised cosine), and $h=\{4 / 16,5 / 16\}$. This is the Advanced Range Telemetry (ARTM) CPM waveform for aeronautical telemetry defined in IRIG 106-04 [17], [18]. The MLSD trellis has 256 states in this case, which is a considerable number. The performance of the optimal detector is given by (31) using the parameters in the first entry in Table I and is plotted in Fig. 3.

The exact PAM representation requires the large set of $48 \times 2$ pulses shown in Fig. 1. We obtain a much smaller set of three pulses by taking the following steps: 1) we apply the minimum mean-squared error approximation in [12] to obtain one averaged set of 12 pulses (this results in an effective value of $L^{\prime}=2$ ) and 2) we further reduce the number of MFs by combining the two length-3T pulses to form one averaged pulse and repeat this for the nine length-2T pulses. This gives the final filter bank of three MFs (these steps are explained in detail in [14]). With $L^{\prime}=2$, the 64-state trellis has branches defined by

$$
\sigma_{n}^{\prime}=\left(\theta_{n-2}, \alpha_{n-1}, \alpha_{n}\right) \text {. }
$$

We must now compute (28) for all of the $\left(\boldsymbol{\alpha}_{i}, \boldsymbol{\alpha}_{j}\right)$ pairs within each merger $\gamma$. The search in (34) finds the same minimum-distance merger for the PAM case as was found for the MLSD case, namely, the first entry in Table I. Technically speaking, with $L=3$, the distance metric $d_{1}(i, j)$ in (29) is a function of the two symbols preceding the merge and the three symbols following the merge; however, the dependence is strongest only on the symbol immediately preceding and immediately following the merge. The result is that we must consider all of the $\left(\boldsymbol{\alpha}_{i}, \boldsymbol{\alpha}_{j}\right)$ 
pairs with $\boldsymbol{\gamma}_{\min }=[0,2,-4,6,-4,2,0]$, where we have explicitly padded a zero coordinate on each end of $\boldsymbol{\gamma}_{\text {min }}$ which may not be ignored when counting the number of terms in (33) (there are 1152 sequence pairs of this type). The squared distances range from 1.03 to 1.52 over these sequence pairs, which is a distance loss of $1.02 \mathrm{~dB}$ for certain transmitted $\alpha_{i}$ sequences. By contrast, the bounding technique in [2] essentially fails by upper bounding the distance loss at $27.24 \mathrm{~dB}$. Fig. 3 shows $P_{b}$ for the PAM-based detector where we see that the bound in (35) becomes indistinguishable from data points obtained by computer simulation as $E_{b} / N_{0}$ grows large.

We also point out that Fig. 3 shows an actual loss of only $0.17 \mathrm{~dB}$ for the PAM-based detector at $P_{b}=10^{-5}$, which is much less than the $1.02 \mathrm{~dB}$ loss of the minimum-distance term in (35); this underscores the point that the actual minimum distance for PAM-based detectors (or all mismatched CPM detectors, for that matter) typically does not have enough weight to approximate $P_{b}$ well for $E_{b} / N_{0}$ of practical interest.

\section{B. Quaternary $2 R C$ With $h=1 / 4$}

The second scheme we consider is $M=4,2 \mathrm{RC}$ with $h=$ $1 / 4$, where the optimal trellis has 16 states. The merger parameters for the MLSD scheme are given in the second entry in Table I.

We consider the PAM configuration for this scheme given in [11] with $L^{\prime}=1$, which has only two pulses and a four-state trellis with branches defined by

$$
\sigma_{n}^{\prime}=\left(\theta_{n-1}, \alpha_{n}\right)
$$

Since $L=2$, the dependence of $d_{1}(i, j)$ on the symbols preceding and following the merger is small and can be ignored in this case. We evaluate (28) for the $18\left(\boldsymbol{\alpha}_{i}, \boldsymbol{\alpha}_{j}\right)$ pairs of interest and obtain squared distances ranging from 1.16 to 1.51 , which is a maximum distance loss of $0.59 \mathrm{~dB}$ (though the loss is much smaller at $P_{b}=10^{-5}$ ). By contrast, the distance bounding technique in [2] yields a less useful upper bound of 1.38-dB loss. Fig. 4 shows $P_{b}$ for this PAM-based detector, which again shows strong agreement with computer simulations as $E_{b} / N_{0}$ grows large.

\section{Binary GMSK With $L=4$ and $B T=1 / 4$}

The last example we consider is binary Gaussian minimumshift keying (GMSK) with $L=4$ and $B T=1 / 4$, which was the central example in [2]. The optimal trellis has 16 states, and the last entry in Table I gives the merger parameters for the optimal detector.

The exact PAM representation for this scheme contains eight pulses with durations of $5 T$ down to $T$. We select approximate PAM-based detectors with two different configurations. The first uses the two most significant pulses, $g_{0}(t)$ and $g_{1}(t)$, as MFs. This makes $L^{\prime}=2$ and produces a four-state trellis with branches given by

$$
\sigma_{n}^{\prime}=\left(\theta_{n-2}, \alpha_{n-1}, \alpha_{n}\right)
$$

This configuration was considered at length in [2]. The second configuration uses only the most significant pulse, $g_{0}(t)$, as an

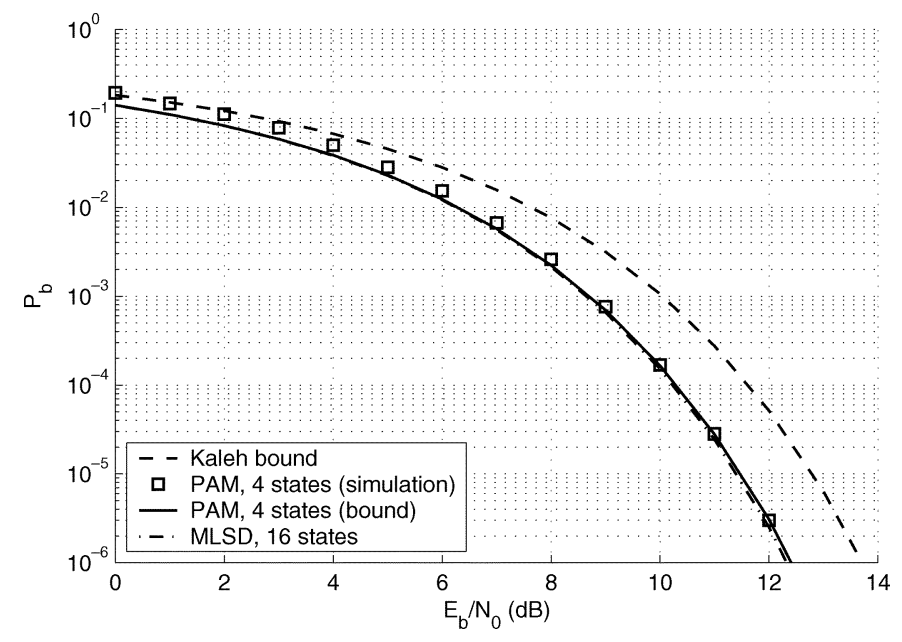

Fig. 4. Performance of 4-ary $2 \mathrm{RC}$ with $h=1 / 4$. The four-state PAM detector has a negligible loss with respect to MLSD. The Kaleh bound [2] is also shown for reference (dashed line).

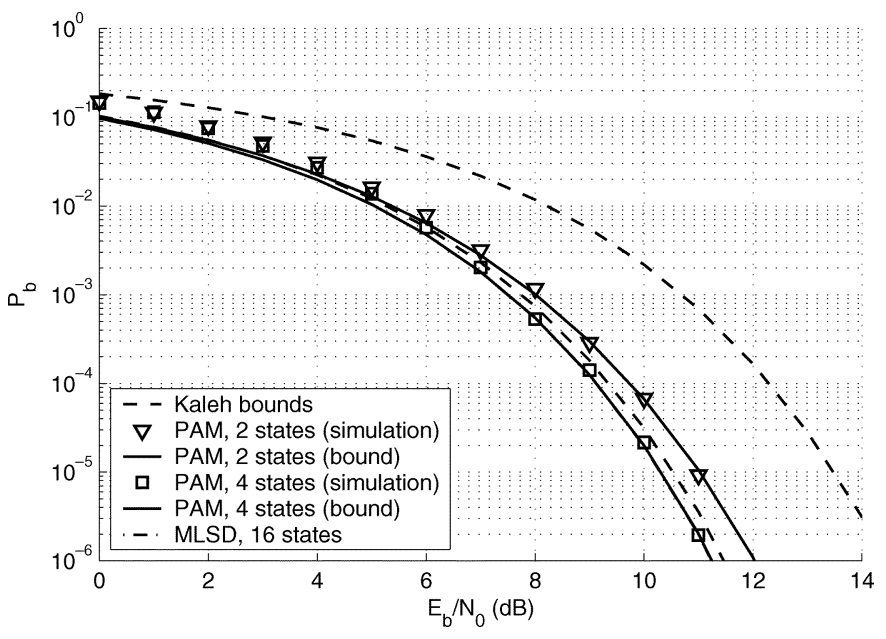

Fig. 5. Performance of binary GMSK with $L=4$ and $B T=1 / 4$. The $P_{b}$ curves show strong agreement with the simulated data points for the two PAM-based detectors (four- and two-states). The bound from Kaleh [2] is also shown for both cases (dashed lines) but it is not tight for the two-state case.

MF. This gives $L^{\prime}=1$ and produces a two-state trellis with branches defined by

$$
\sigma_{n}^{\prime}=\left(\theta_{n-1}, \alpha_{n}\right)
$$

As with the first example, we must pad a zero coordinate on each end of the $\gamma$ sequences when performing the search in (34). For the four-state detector, the squared distances range from 1.66 to 1.72 . The analysis in [2] upper bounds this distance loss at $0.24 \mathrm{~dB}$. For the second detector configuration (two states), the squared distances range from 1.29 to 2.07 and the distance bound from [2] is less helpful at 3.18-dB loss. The $P_{b}$ curves generated by (35) for the two PAM configurations are shown in Fig. 5 along with data from computer simulations.

\section{APPLICATIONS}

Given that the reduced-complexity detectors in [1], [6], and Section II each gather distance in their own unique manner while using the exact same trellis, an interesting question to ask is: which method yields the best performance for a given trellis 
TABLE II

PERFORMANCE OF REDUCED-COMPLEXITY DETECTORS FOR $M=4,3 \mathrm{RC}$, $h=\{4 / 16,5 / 16\}$

\begin{tabular}{c|c|c|c}
\hline Approximation & States & $d_{\min }^{\prime 2}(i, j)$ & $\begin{array}{l}\mathrm{dB} \text { loss } \\
\text { at } P_{b}= \\
10^{-5}\end{array}$ \\
\hline MLSD, $L=3, p=16$ & 256 & 1.29 & - \\
\hline \hline PAM, $L^{\prime}=2, p=16$ & 64 & 1.03 & 0.17 \\
\hline$[1], L^{\prime}=2, p=16$ & 64 & 1.17 & 0.04 \\
\hline$[6], L^{\prime}=2, p=16$ & 64 & 1.27 & 0.07 \\
\hline \hline PAM, $L^{\prime}=1, p=16$ & 16 & 0.44 & 1.6 \\
\hline$[1], L^{\prime}=1, p=16$ & 16 & 0.31 & 3.8 \\
\hline$[6], L^{\prime}=1, p=16$ & 16 & 0.92 & 1.5 \\
\hline \hline PAM, $L^{\prime}=2, p^{\prime}=4$ & 16 & 1.03 & 0.9 \\
\hline$[1], L^{\prime}=2, p^{\prime}=4$ & 16 & 1.17 & 1.2 \\
\hline$[6], L^{\prime}=2, p^{\prime}=4$ & 16 & 0.59 & 3.4 \\
\hline
\end{tabular}

complexity? It comes as no surprise that the answer depends on the CPM scheme and the trellis in question. In this section we demonstrate the typical characteristics of each detection scheme, using the multi- $h$ scheme $M=4,3 \mathrm{RC}, h=$ $\{4 / 16,5 / 16\}$ as a case study. We will show that the PAM-based detector performs well in cases where the trellis size is aggressively reduced. This is due primarily to the different manner in which distance accumulates in PAM-based detectors.

In Table II we consider three reduced trellis configurations. There are three groupings in the table, one for each configuration. The first entry in the table is for the MLSD scheme which serves as the reference detector for what follows.

The first reduced trellis is the 64-state configuration with branches defined by (36). This is a relatively minor state reduction of a factor of 4 . The first grouping in Table II shows that the three reduced complexity detectors perform relatively close to one another, with the PAM-based detector being slightly the worst of the three both in terms of minimum distance and loss at $P_{b}=10^{-5}$. In all of these detectors, the minimum-distance merger is the one given for this CPM scheme in Table I. The detector from [1] is based on a 2RC approximation. Its minor losses are the result of this transmitter/receiver mismatch and from the fact that $D_{\text {merger }}$ is shortened from seven to six symbol times. As mentioned before, the one symbol time worth of distance loss is divided equally between the beginning and ending tail of the error event. The detector from [6] uses decision feedback to approximate the symbol $\alpha_{n-2}$ which is found in the original 4-tuple in (7). Its minor loss is entirely due to the shortening of $D_{\text {merger }}$. In this case, it forfeits the distance increment that might have come in the seventh symbol time of the error event, namely, the small increment $1.29-1.27=0.02$. The PAM-based detector is the same as given in Section IV-A, which uses three pulses to approximate the original set of $48 \times 2$. Its third-place ranking is balanced by the fact that its filtering requirements are much less than the other two detectors.

We can pursue more aggressive trellis approximations. The second reduced trellis we consider has branches defined by

$$
\sigma_{n}^{\prime}=\left(\theta_{n-1}, \alpha_{n}\right)
$$

which is a trellis of 16 states (a state reduction by a factor of 16). To achieve this reduction, the detector from [1] is based on a $1 \mathrm{RC}$ approximation, and the detector from [6] uses decision feedback to approximate the symbols $\alpha_{n-2}$ and $\alpha_{n-1}$ that are found in the original 4-tuple in (7). The PAM-based detector uses the $3 \times 2$ pulses from the minimum mean-squared error approximation in [12]. The performance of these three detectors is shown in the second grouping in Table II. Here the decision feedback approach from [6] and the PAM-based detector perform close to one another, while the detector from [1] suffers a large loss due to the coarseness of the 1RC approximation.

For the third reduced trellis example, we use decision feedback to reduce the number of phase states from $p=16$ to $p^{\prime}=4$, cf. e.g., [6]. The branches in this 16-state trellis are defined by

$$
\sigma_{n}^{\prime}=\left(\left(\theta_{n-2}\right)_{\bmod } 4, \alpha_{n-1}, \alpha_{n}\right)
$$

where the modulo-4 operation in (41) is understood to apply to the modulo- $p$ operation in (6). We use the same three detector approximations as in the 64-state case, namely the 2RC mismatch, the $\alpha_{n-2}$ decision feedback and the three-pulse PAM approximation. The benefit of the $p^{\prime}=4$ trellis is an overall reduction in states by a factor of 16 , which gives the same number of states as the previous trellis example, although it is an entirely different trellis. The downside of the approximation is that this new trellis definition permits mergers according to (32) using $p^{\prime}=4$ instead of $p=16$. The most potentially catastrophic of these is $\boldsymbol{\gamma}_{1}=\ldots, 0,2,0, \ldots$, where $D_{\text {merger }}=2$ symbol times.

The last grouping in Table II shows the performance of the three detectors using this trellis approximation. In this example the detector from [6] suffers greatly because the $\boldsymbol{\gamma}_{1}$ merger is dominant, where squared distance is only 0.31 after two symbol times. This is unfortunate, because after the second symbol time the distance grows rather quickly (and indefinitely) with a linear slope, since the data sequences represented by $\boldsymbol{\gamma}_{1}$ produce CPM signals which remain different forever. On the other hand, the mismatched detector from [1] benefits greatly from the extra half symbol time it gets to observe the $\boldsymbol{\gamma}_{1}$ merger. The squared distances for this merger range from 1.32 to 1.51 , with a somewhat uniform distribution. Therefore, the minimum distance for this detector in Table II is unchanged from the 64-state example. However, these near-minimum-distance mergers have a high probability of transmission and produce a 1.2-dB loss for this detector at $P_{b}=10^{-5}$. The PAM-based detector also benefits from its different observation of the $\gamma_{1}$ merger. Here the squared distances range from 1.20 to 5.29 , which is a large spread with most of the terms at the high end. The minimum distance for the PAM-based detector is also unchanged from the 64-state example. Its smaller loss of $0.9 \mathrm{~dB}$ at $P_{b}=10^{-5}$ is due to the larger distance of most of the $\boldsymbol{\gamma}_{1}$ terms.

Although we have focused on only one CPM scheme in this study, the results given are typical for these detectors (see also [1], [2], [6], and [11]). As $L^{\prime}$ is decreased, the detector from [1] suffers the most due to the coarseness of its mismatch with the transmitter. As $p^{\prime}$ is decreased, the decision feedback detector from [6] suffers the most due to the rogue mergers permitted by the reduced trellis. In either case, PAM-based detectors sustain relatively manageable losses. 


\section{CONCLUSION}

We have presented the form of the optimal and approximate detectors for multi- $h$ CPM which are based on the recently extended PAM representation of multi- $h$ CPM. We have also found an exact expression for the pairwise error probability for all PAM-based CPM detectors. This expression was used to evaluate the performance of detectors for single- and multi- $h$ CPM, where there was strong agreement with computer simulations and where a previously reported performance bounding technique was found to be less useful. It was shown that the key performance characteristic of these detectors is that their metrics observe the received signal for longer durations than those of other reduced-complexity detectors for CPM. This permits a very aggressive application of trellis reduction techniques, parameterized by $p^{\prime}$ and $L^{\prime}$, with relatively minor performance losses. This result unifies and confirms those reported by other authors which were obtained by computer simulations. It was also shown that detectors with traditional metrics can suffer great losses when similarly aggressive trellis reductions were applied.

\section{REFERENCES}

[1] A. Svensson, C.-E. Sundberg, and T. Aulin, "A class of reduced-complexity Viterbi detectors for partial response continuous phase modulation," IEEE Trans. Commun., vol. 32, no. 10, pp. 1079-1087, Oct. 1984.

[2] G. K. Kaleh, "Simple coherent receivers for partial response continuous phase modulation," IEEE J. Sel. Areas Commun., vol. 7, no. 12, pp. 1427-1436, Dec. 1989.

[3] J. Huber and W. Liu, "An alternative approach to reduced complexity CPM receivers," IEEE J. Sel. Areas Commun., vol. 7, no. 12, pp. 1427-1436, Dec. 1989.

[4] P. Moqvist and T. Aulin, "Orthogonalization by principal components applied to CPM,’ IEEE Trans. Commun., vol. 51, no. 11, pp. 1838-1845, Nov. 2003.

[5] T. Aulin, "Study of a New Trellis Decoding Algorithm and its Applications," European Space Agency, Noordwijk, The Netherlands, ESTEC Contract 6039/84/NL/DG, 1985.

[6] A. Svensson, "Reduced state sequence detection of partial responce continuous phase modulation," Proc. Inst. Elect. Eng., pt. I, vol. 138, pp. 256-268, Aug. 1991.

[7] S. J. Simmons and P. H. Wittke, "Low complexity decoders for constant envelope digital modulations," IEEE Trans. Commun., vol. COM-31, no. 12, pp. 1273-1280, Dec. 1983.

[8] P. A. Laurent, "Exact and approximate construction of digital phase modulations by superposition of amplitude modulated pulses (AMP)," IEEE Trans. Commun., vol. COM-34, no. 2, pp. 150-160, Feb. 1986.

[9] U. Mengali and M. Morelli, "Decomposition of $M$-ary CPM signals into PAM waveforms," IEEE Trans. Inf. Theory, vol. 41, no. 9, pp. 1265-1275, Sep. 1995.

[10] X. Huang and Y. Li, "The PAM decomposition of CPM signals with integer modulation index," IEEE Trans. Commun., vol. 51, no. 4, pp. 543-546, Apr. 2003.

[11] G. Colavolpe and R. Raheli, "Reduced-complexity detection and phase synchronization of CPM signals," IEEE Trans. Commun., vol. 45, no. 9, pp. 1070-1079, Sep. 1997.
[12] E. Perrins and M. Rice, "PAM decomposition of $M$-ary multi- $h$ CPM," IEEE Trans. Commun., to be published.

[13] B. E. Rimoldi, "A decomposition approach to CPM," IEEE Trans. Inf. Theory, vol. 34, no. 2, pp. 260-270, Mar. 1988.

[14] E. Perrins and M. Rice, "Optimal and reduced complexity receivers for M-ary multi-h CPM," in Proc. IEEE Wireless Commun. Netw. Conf., Atlanta, GA, Mar. 2004, pp. 1165-1170.

[15] J. B. Anderson, T. Aulin, and C.-E. Sundberg, Digital Phase Modulation. New York: Plenum, 1986.

[16] P. Moqvist and T. Aulin, "Serially concatenated continuous phase modulation with iterative decoding," IEEE Trans. Commun., vol. 49, no. 11, pp. 1901-1915, Nov. 2001.

[17] M. Geoghegan, "Description and performance results for a multi-h CPM telemetry waveform," in Proc. IEEE MILCOM, vol. 1, Oct. 2000, pp. 353-357.

[18] (2004) IRIG Standard 106-04: Telemetry Standards. Range Commanders Council Telemetry Group, Range Commanders Council, White Sands Missile Range, White Sands, NM. [Online]. Available: http://jcs.mil/RCC/manuals/106-04

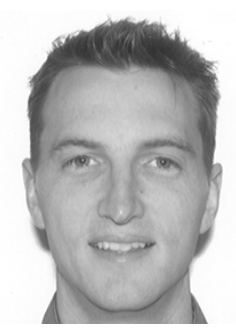

Erik Perrins (S'96-M'05) received the B.S. (magna cum laude), M.S., and Ph.D. degrees from Brigham Young University, Provo, UT, in 1997, 1998, and 2005, respectively.

From 1998 to 2004, he was with Motorola, Inc., Schaumburg, IL, where he was involved with advanced development of land mobile radio products. Since 2004, he has been an industry consultant on receiver design problems such as synchronization and complexity reduction. He joined the faculty of the Department of Electrical Engineering and Computer Science, University of Kansas, Lawrence, in August 2005. His research interests are in digital communication theory, synchronization, channel coding, and complexity reduction in receivers.

Prof. Perrins is a member of the IEEE Communications Society.

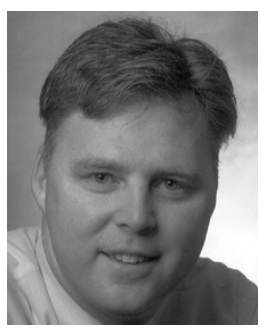

Michael Rice (M'82-SM'98) received the B.S.E.E. degree from Louisiana Tech University, Ruston, in 1987 and the Ph.D. degree from the Georgia Institute of Technology, Atlanta, in 1991.

He was with Digital Transmission Systems, Inc., Atlanta, and joined the faculty at Brigham Young University, Provo, UT, in 1991 where he is currently the Jim Abrams Professor with the Department of Electrical and Computer Engineering. He was a NASA/ASEE Summer Faculty Fellow with the Jet Propulsion Laboratory during 1994 and 1995, where he was involved with land mobile satellite systems. During the 1999-2000 academic year, he was a Visiting Scholar with the Communication Systems and Signal Processing Institute, San Diego State University, San Diego, CA. His research interests are in the area of digital communication theory and error control coding, with a special interest in applications to telemetering and software radio design. He has been a consultant to both government and industry on telemetry related issues.

Prof. Rice is a member of the IEEE Communications Society. He was Chair of the Utah Section of the IEEE from 1997 to 1999 and Chair of the Signal Processing and Communications Society Chapter of the Utah Section from 2002 to 2003 . 